\title{
Four-vertex Model and Random Tilings
}

\author{
N.M. Bogoliubov \\ St. Petersburg Department of V.A. Steklov Mathematical Institute, \\ 27, Fontanka, St. Petersburg 191023, Russia
}

\begin{abstract}
The exactly solvable four-vertex model on a square grid with the different boundary conditions is considered. The application of the Algebraic Bethe Ansatz method allows to calculate the partition function of the model. For the fixed boundary conditions the connection of the scalar product of the state vectors with the generating function of the column and row strict boxed plane partitions is established. Tiling model on a periodic grid is discussed.
\end{abstract}

\section{INTRODUCTION}

The connection of integrable models with the enumerative combinatorics is widely discussed. The six-vertex model plays an important role in these considerations. The model was widely studied both for periodic boundary conditions and for the fixed ones [1, 2, 3, 4, 5, 6 ]. The six-vertex model for the so-called fixed boundary conditions is related to the enumeration of domino tilings of Aztec diamonds and of alternating sign matrices [7, 8, 9].

The four-vertex model is a particular case of the six-vertex model in which two vertices are frozen out. This model was considered in [10, 11] where the connection of this model with a random tiling models on a semi-infinite strip geometry was discussed. Tiling models are classical discrete statistical models in which the tiles of the different geometric shapes are packed together so that they cover space without holes or overlaps. They are called random only to emphasize the difference with perfect quasi-periodic tilings.

In this paper we shall solve the model by the direct application of the Quantum Inverse Scattering Method (QISM) [12, 13]. This approach will allow us to study the model on a finite lattice with the different boundary conditions and to calculate partition function of both vertex and corresponding tiling models. In particular, we shall show that the partition function of the four-vertex model with the fixed boundary conditions is related to the generating function of the boxed plane partitions. The boxed plane partitions are equivalent to the three dimensional Young diagrams placed into a box of a finite size and to lozenge tiling 
of an semiregular hexagon [14, 15]. For the periodic boundary conditions the four-vertex model is equivalent to the lozenge tiling of the torus.

The connection of the boson type integrable models with the theory of symmetric functions 14] and with the theory of plane partitions [16] was considered in the papers [17, 18, 19, 20].

The paper is organized as follows. We give the definition of the model and discuss the QISM approach to its solution in Sec. II. In Sec. III the model is studied on the lattice with the fixed boundary conditions and its connection with the plane partitions is established. The partition function of the model on the periodic grid is calculated in Sec. IV.

\section{FOUR-VERTEX MODEL}

A special case of the six vertex model - a four vertex model on a square grid is described by four different arrows arrangements pointing in and out of each vertex. A statistical weight corresponds to each type of the vertices and there are three vertex weights $\omega_{a}, \omega_{b}$ and $\omega_{c}$ (FIG. 1). There is an alternate description of the vertices in terms of lines flowing through the vertices. Since a lattice edge can exist in two states, line or no line, there exists a one-to-one correspondence between the arrow configuration on the lattice and the graphs of lines on the lattice - nests of lattice paths.

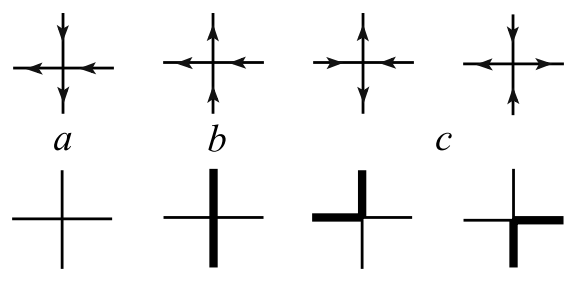

FIG. 1: The four allowed types of vertices in terms of arrows and lines.

For the homogeneous model the vertex weights do not depend on the position of the vertex and the partition function is equal to

$$
Z\left(\omega_{a}, \omega_{b}, \omega_{c}\right)=\sum \omega_{a}^{l^{a}} \omega_{b}^{l^{b}} \omega_{c}^{l^{c}}
$$

where the summation is extended over all allowed configurations of the arrows on a lattice. These configurations depend on the imposed boundary conditions which are specified by the direction of the arrows on the boundary of the grid. The number of vertices $(a),(b),(c)$ 
in each configuration is $l^{a}, l^{b}, l^{c}$. For the inhomogeneous model the vertex weights are site dependent.

To apply the Quantum Inverse Scattering Method we use the spin description of the model. With each vertical bond of the grid and horizontal bond one associates the space $\mathcal{C}^{2}$, with spin up and spin down states forming a natural basis in this space. The spin up state on the vertical bond corresponds to the line pointing up, while the spin down state to the line pointing down. The spin up state on the $i$-th horizontal bond $\left(\begin{array}{l}1 \\ 0\end{array}\right)_{i} \equiv|\leftarrow\rangle_{i}$ corresponds to the horizontal line pointing to the left, spin down state to the line pointing to the right $\left(\begin{array}{l}0 \\ 1\end{array}\right)_{i} \equiv|\rightarrow\rangle_{i}$. The total space of the vertical lines is $\mathcal{V}=\left(\mathcal{C}^{2}\right)^{\otimes N}$ and we shall call it an auxiliary space. The total space of the horizontal lines is $\mathcal{H}=\left(\mathcal{C}^{2}\right)^{\otimes N}$, and we shall call it a quantum space. With each vertex of the lattice one associates an operator acting in the full space $\mathcal{V} \otimes \mathcal{H}$. This operator is called $L$-operator and it acts nontrivially only in a single vertical space $\mathcal{C}^{2}$ and in single horizontal space $\mathcal{C}^{2}$, while in all other spaces it acts as the unity operator.

The $L$-operator of the four vertex model is equal to

$$
L(n \mid u)=-\frac{u}{2}\left(1+\sigma^{z}\right) e_{n}+\frac{u^{-1}}{2}\left(1-\sigma^{z}\right) e_{n}+\sigma^{+} \sigma_{n}^{-}+\sigma^{-} \sigma_{n}^{+},
$$

where $\sigma^{z, \pm}$ - are the Pauli matrices, the projector $e=\frac{1}{2}\left(\sigma^{z}+1\right)$, and the matrix with subindex $n$ acts nontrivially only in the $n$-th space: $s_{n}=I \otimes \ldots \otimes I \otimes s \otimes I \otimes \ldots \otimes I$.

One can represent the matrix elements of the introduced $L$-operator

$$
L(n \mid u)=\left(\begin{array}{cc}
L_{11}(n \mid u) & L_{12}(n \mid u) \\
L_{21}(n \mid u) & L_{22}(n \mid u)
\end{array}\right)=\left(\begin{array}{cc}
-u e_{n} & \sigma_{n}^{-} \\
\sigma_{n}^{+} & u^{-1} e_{n}
\end{array}\right)
$$

as a dot with the attached arrows (see FIG. 2). The matrix element $L_{11}(n \mid u)$ corresponds to a

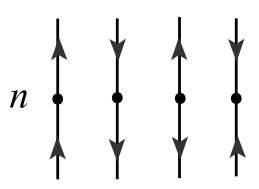

(1) (2) (3) (4)

FIG. 2: Vertex representation of the matrix elements of the $L$-operator.

vertex (1) (FIG. 2), where a dot stands for the operator $-u e_{n}$, this operator acts on the local 
spin state and the only non-zero matrix element of this operator is ${ }_{n}\left\langle\leftarrow\left|-u e_{n}\right| \leftarrow\right\rangle_{n}$ what gives the vertex (b) (FIG. 1) with a weight $-u$. The matrix element $L_{22}(n \mid u)$ corresponds to a vertex (2) (FIG. 2), where a dot is the operator $u^{-1} e_{n}$ and the non-zero matrix element ${ }_{n}\left\langle\leftarrow\left|u^{-1} e_{n}\right| \leftarrow\right\rangle_{n}$ is the vertex (a) (FIG. 1) with a weight $u^{-1}$. The matrix elements $L_{12}(n \mid u)$, and $L_{21}(n \mid u)$ correspond to the vertices (3) and (4) (FIG. 2) respectively, and the nonzero matrix elements ${ }_{n}\left\langle\rightarrow\left|\sigma_{n}^{-}\right| \leftarrow\right\rangle_{n}$ and ${ }_{n}\left\langle\leftarrow\left|\sigma_{n}^{+}\right| \rightarrow\right\rangle_{n}$ are the vertices (c) (FIG. 1) with a weight 1.

The $L$-operator (2) satisfies the intertwining relation

$$
R(u, v)(L(n \mid u) \otimes L(n \mid v))=(L(n \mid v) \otimes L(n \mid u)) R(u, v),
$$

in which $R(u, v)$ is the $4 \times 4$ matrix

$$
R(u, v)=\left(\begin{array}{cccc}
f(v, u) & 0 & 0 & 0 \\
0 & g(v, u) & 1 & 0 \\
0 & 0 & g(v, u) & 0 \\
0 & 0 & 0 & f(v, u)
\end{array}\right)
$$

with

$$
f(v, u)=\frac{u^{2}}{u^{2}-v^{2}}, g(v, u)=\frac{u v}{u^{2}-v^{2}} .
$$

The vertical monodromy matrix is the product of $L$-operators

$$
T(u)=L(M \mid u) L(M-1 \mid u) \ldots L(0 \mid u)=\left(\begin{array}{cc}
A(u) & B(u) \\
C(u) & D(u)
\end{array}\right) .
$$

The matrix entries of the monodromy matrix (7) are expressed as sums over all possible configurations of arrows with different boundary conditions on a one-dimensional lattice with $M+1$ sites (FIG. 3). Namely, operator $B(u)=\sum_{k_{M}, \ldots, k_{1}=1}^{2} L_{1 k_{M}}(M \mid u) L_{k_{M} k_{M-1}}(M-$ $1 \mid u) \ldots L_{k_{1} 2}(0 \mid u)$ corresponds to the boundary conditions when arrows on the top and bottom of the lattice are pointing outwards (configuration (B)). Operator $C(u)$ corresponds to the boundary conditions when arrows on the top and bottom of the lattice are pointing inward (configuration $(\mathrm{C})$ ). Operators $A(u)$ and $D(u)$ correspond to the boundary conditions when arrows on the top and bottom of the lattice are pointing up and down respectively (configurations (A) and (D)).

The commutation relations of the matrix elements of the monodromy matrix are given by the same $R$-matrix (5)

$$
R(u, v)(T(u) \otimes T(v))=(T(v) \otimes T(u)) R(u, v) .
$$




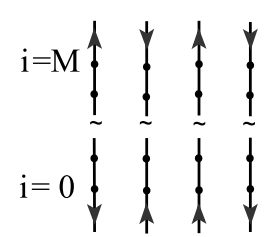

(B) (C) (A) (D)

FIG. 3: Graphic representation of the entries of the monodromy matrix

The most important relations are

$$
\begin{aligned}
C(u) B(v) & =g(u, v)\{A(u) D(v)-A(v) D(u)\}, \\
A(u) B(v) & =f(u, v) B(v) A(u)+g(v, u) B(u) A(v), \\
D(u) B(v) & =f(v, u) B(v) D(u)+g(u, v) B(u) D(v), \\
{[B(u), B(v)] } & =[C(u), C(v)]=0 .
\end{aligned}
$$

The transfer matrix $\tau(u)$ is the trace of the monodromy matrix in the auxiliary space

$$
\tau(u)=\operatorname{tr} T(u)=A(u)+D(u)
$$

The relation (8) means that $[\tau(u), \tau(v)]=0$ for arbitrary values of parameters $u, v$.

The $L$-operator (2) satisfies the relation

$$
e^{\zeta \sigma_{n}^{z}} L(n \mid u) e^{-\zeta \sigma_{n}^{z}}=e^{-\frac{\zeta}{2} \sigma^{z}} L(n \mid u) e^{\frac{\zeta}{2} \sigma^{z}},
$$

where $\zeta$ is an arbitrary parameter. From this equation and from the definition of the monodromy matrix (7) it follows that

$$
e^{\zeta S^{z}} T(u) e^{-\zeta S^{z}}=e^{-\zeta \sigma^{z}} T(u) e^{\zeta \sigma^{z}}
$$

where $S^{z}=\frac{1}{2} \sum_{i=0}^{M} \sigma_{i}^{z}$ is the operator of $z$ component of the total spin. The equalities

$$
\begin{aligned}
& S^{z} B(u)=B(u)\left(S^{z}-1\right), \\
& S^{z} C(u)=C(u)\left(S^{z}+1\right),
\end{aligned}
$$

are the consequence of the equation (12). It means that the operator $B(u)$ decreases the total spin of the system, while $C(u)$ increases it. From the equation (12) follows the commutativity

$$
\left[\tau(u), S^{z}\right]=0
$$


The generating state in the space $\mathcal{H}$ is the state with all spins up

$$
|\Leftarrow\rangle=\otimes_{i=0}^{M}|\leftarrow\rangle_{i}=\otimes_{i=0}^{M}\left(\begin{array}{l}
1 \\
0
\end{array}\right)_{i}
$$

It is annihilated by the operator $C(u)$

$$
C(u)|\Leftarrow\rangle=0
$$

and it is an eigenvector of operators $A(u)$ and $D(u)$,

$$
A(u)|\Leftarrow\rangle=\alpha(u)|\Leftarrow\rangle ; \quad D(u)|\Leftarrow\rangle=\delta(u)|\Leftarrow\rangle,
$$

with the eigenvalues

$$
\alpha(u)=(i u)^{M+1}, \quad \delta(u)=\left(\frac{i}{u}\right)^{M+1} .
$$

The total spin of the generating state is $\frac{1}{2}(M+1)$ :

$$
S^{z}|\Leftarrow\rangle=\frac{1}{2}(M+1)|\Leftarrow\rangle .
$$

One of the main objects in the further consideration will be the vector generated by the multiple action of operators $B(u)$ on the state $|\Leftarrow\rangle$

$$
\left|\Psi_{n}\left(u_{1}, u_{2}, \ldots, u_{N}\right)\right\rangle=\prod_{i=1}^{N} B\left(u_{i}\right)|\Leftarrow\rangle .
$$

From (13) it follows that the total spin of this vector is

$$
S^{z} \prod_{i=1}^{N} B\left(u_{i}\right)|\Leftarrow\rangle=\frac{1}{2}(M+1-2 N) \prod_{i=1}^{N} B\left(u_{i}\right)|\Leftarrow\rangle .
$$

The state conjugated to (20) is

$$
\left\langle\Psi_{n}\left(u_{1}, u_{2}, \ldots, u_{N}\right)\right|=\langle\Leftarrow| \prod_{i=1}^{N} C\left(u_{i}\right) .
$$

It is easy to verify that $\langle\Leftarrow| B(u)=0$.

In the coordinate representation the state vector takes the form

$$
\left|\Psi_{N}\left(u_{1}, u_{2}, \ldots, u_{N}\right)\right\rangle=\prod_{k=1}^{N} B\left(u_{k}\right)|\Leftarrow\rangle=\sum \chi_{\mu}\left(u_{1}, u_{2}, \ldots, u_{N}\right)\left|m_{1}, \ldots, m_{n}\right\rangle .
$$

Here we denote the state with $N$ spins down in the sites $m_{1}, \ldots, m_{N}$ and with $M+1-N$ spins up in the other sites by $\left|m_{1}, \ldots, m_{N}\right\rangle$. The wave function $\chi_{\mu}\left(u_{1}, u_{2}, \ldots, u_{N}\right)$ satisfies the 
exclusion condition. It is non equal to zero only if numbers $\mu=\left(m_{1}, m_{2}, \ldots, m_{N}\right)$ form a strict partition $M \geq m_{1}>m_{2}>\ldots>m_{N} \geq 0$ and $m_{i} \geq m_{i+1}+2$. By the direct calculations it may be shown that the wave function is of determinantal form:

$$
\chi_{\mu}\left(u_{1}, u_{2}, \ldots, u_{N}\right)=(-1)^{\sum m_{k}}\left(u_{1} \ldots u_{N}\right)^{M-2(N-1)} \frac{\operatorname{det}\left(u_{j}^{-2\left(m_{k}+k-N\right)}\right)}{\prod_{1 \leq k<j \leq N}\left(u_{k}^{-2}-u_{j}^{-2}\right)} .
$$

In the terms of the symmetric Schur function

$$
S_{\lambda}\left(u_{1}, u_{2}, \ldots, u_{N}\right)=\frac{\operatorname{det}\left(u_{j}^{N-k+\lambda_{k}}\right)}{\prod_{1 \leq k<j \leq N}\left(u_{k}-u_{j}\right)},
$$

it has the form

$$
\chi_{\mu}\left(u_{1}, u_{2}, \ldots, u_{N}\right)=(-1)^{\sum m_{k}}\left(u_{1} \ldots u_{N}\right)^{M-2(N-1)} S_{\mu-\delta}\left(u_{1}^{-2}, u_{2}^{-2}, \ldots, u_{N}^{-2}\right),
$$

where $\delta$ is a partition with the elements $\delta_{j}=2(N-j), j=1, \ldots, N$, and the elements of the partition $\lambda=\mu-\delta$ are $\lambda_{j}=m_{j}-2(N-j)$ satisfy the condition $M-2(N-1) \geq \lambda_{1} \geq$ $\lambda_{2} \geq \ldots \geq \lambda_{N} \geq 0$.

\section{FIXED BOUNDARY CONDITIONS}

Let us consider now a model on a $2 N \times(M+1)$ square lattice with a following boundary conditions: all arrows on the left and right boundaries are pointing to the left while the arrows on the top and bottom of the first $N$ columns (counting from the left) are pointing inwards and the arrows on the top and bottom of the last $N$ ones are pointing outwards. We shall call this condition - the fixed boundary condition.

To enumerate all possible configuration of the vertices it is more convenient to use the description of the model in terms of flowing lines and to represent the allowed configurations as the nests of lattice paths. The path is running from one of the $N$ down left vertices to the top $N$ right ones and always moves east or north. The paths cannot touch each other and arbitrary number of consequent steps are allowed in vertical direction while only one step at a time is allowed in the horizontal one. If the first $N$ columns have the numbers $(-N, \ldots,-1)$ and the last ones $(1, \ldots, N)$, if the lower row has the number 0 and the upper one $M$, then the $m$-th path is running from the vertex $(-N+m-1 ; 0)$ to the vertex $(m ; M)$, $1 \leq m \leq N$. A typical nest of lattice paths is represented in (FIG. 4). The length of the path is $N+M$. The number of the $(c)$ vertices in the admissible path is $2 N$ since only one step is 
allowed in horizontal direction, so the number of $(b)$ vertices is $M-N+1$. It means that the number of $(c)$ and $(b)$ vertices in the nest is equal to $l^{c}=2 N^{2}$ and $l^{b}=l^{a}=N(M-N+1)$ respectively.
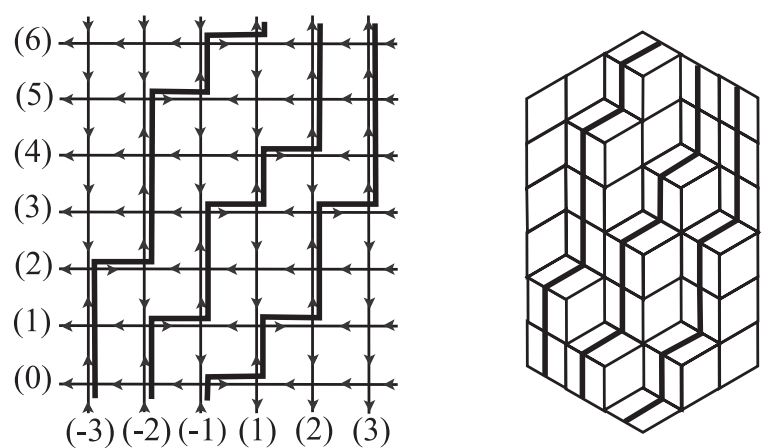

FIG. 4: A typical configuration of admissible lattice paths with the fixed boundary conditions and the corresponding plane partition with the gradient lines.

The partition function of the homogenous model with the fixed boundary conditions is equal to

$$
Z\left(\omega_{a}, \omega_{b}, \omega_{c}\right)=\left(\omega_{a} \omega_{b}\right)^{N(M-N+1)} \omega_{c}^{2 N^{2}} S(N, M)
$$

where $S(N, M)$ is the total number of the allowed nests of lattice paths.

In the inhomogeneous model the vertex weights $\omega_{a}, \omega_{b}$ and $\omega_{c}$ are site dependent. We shall consider a case when the weights depend on the number of the column only. If the first $N$ columns have the numbers $(-N, \ldots,-1)$ and the last ones $(1, \ldots, N)$ then the partition function is equal to

$$
Z\left(\left\{\omega_{a}\right\},\left\{\omega_{b}\right\},\left\{\omega_{c}\right\}\right)=\sum \prod_{k=-1}^{-N}\left(\omega_{a}\right)_{k}^{l_{k}^{a}}\left(\omega_{b}\right)_{k}^{l_{k}^{b}}\left(\omega_{c}\right)_{k}^{l_{k}^{c}} \prod_{j=1}^{N}\left(\omega_{a}\right)_{j}^{l_{j}^{a}}\left(\omega_{b}\right)_{j}^{l_{j}^{b}}\left(\omega_{c}\right)_{j}^{l_{j}^{c}}
$$

Consider the scalar product of the state vectors (201) and (22)

$$
W\left(u_{1}, \ldots, u_{N} ; v_{1}, \ldots, v_{N}\right)=\left\langle\Leftarrow\left|C\left(v_{1}\right) \ldots C\left(v_{N}\right) B\left(u_{1}\right) \ldots B\left(u_{N}\right)\right| \Leftarrow\right\rangle,
$$

where $\{u\}$ and $\{v\}$ are the sets of independent parameters. We can represent the matrix element (28) as the two-dimensional square lattice with $2 N \times(M+1)$ sites. First $N$ vertical rows of the lattice are associated with the operators $C\left(v_{j}\right)$ and the last $N$ vertical rows with operators $B\left(u_{j}\right)$. The horizontal rows of the lattice are associated with the local spin spaces, $i$-th row with the $i$-th space respectively. From the graphical representation of the 
operators $B(u)$ and $C(u)$ it follows that the matrix element (28) is equal to the sum over all allowed configurations of vertices on a square lattice with the arrows on first $N$ vertical rows pointing inwards, on the last $N$ ones pointing outwards; on the right and on the left boundaries all arrows are pointing to the left (FIG. 4):

$$
\begin{aligned}
W\left(u_{1}, \ldots, u_{N} ; v_{1}, \ldots, v_{N}\right) & =\sum \prod_{k=-1}^{-N}\left(-v_{-k}\right)^{l_{k}^{b}}\left(v_{-k}^{-1}\right)^{l_{k}^{a}} \prod_{j=1}^{N}\left(-u_{j}\right)^{l_{j}^{b}}\left(u_{j}^{-1}\right)^{l_{j}^{a}} \\
& =(-1)^{M N} \sum \prod_{k=-1}^{-N} v_{-k}^{l_{k}^{b}-l_{k}^{a}} \prod_{j=1}^{N} u_{j}^{l_{j}^{b}-l_{j}^{a}} .
\end{aligned}
$$

If we put

$$
\begin{aligned}
& \left(\omega_{a}\right)_{j}=v_{-j}^{-1},\left(\omega_{b}\right)_{j}=v_{-j} ;-N \leq j \leq-1, \\
& \left(\omega_{a}\right)_{j}=u_{j}^{-1},\left(\omega_{b}\right)_{j}=u_{j} ; 1 \leq j \leq N, \\
& \left(\omega_{c}\right)_{j}=1,
\end{aligned}
$$

then

$$
Z\left(\left\{\omega_{a}\right\},\left\{\omega_{b}\right\},\left\{\omega_{c}\right\}\right)=(-1)^{M N} W\left(u_{1}, \ldots, u_{N} ; v_{1}, \ldots, v_{N}\right) .
$$

The matrix element (28) for the arbitrary values of the parameters $u_{j}, v_{j}$ is evaluated by means of the commutation relations (9) [13, 17] and is equal to:

$$
W\left(u_{1}, \ldots, u_{n} ; v_{1}, \ldots, v_{n}\right)=(-1)^{M N}\left\{\prod_{j>k} \frac{v_{j} v_{k}}{v_{k}^{2}-v_{j}^{2}} \prod_{l>m} \frac{u_{l} u_{m}}{u_{l}^{2}-u_{m}^{2}}\right\} \operatorname{det} H,
$$

where the entries of $N \times N$ matrix $H$ are

$$
H_{j k}=\left\{\left(\frac{u_{k}}{v_{j}}\right)^{M-N+2}-\left(\frac{u_{k}}{v_{j}}\right)^{-M+N-2}\right\} \times \frac{1}{\frac{u_{k}}{v_{j}}-\left(\frac{u_{k}}{v_{j}}\right)^{-1}} .
$$

Formulas (32), (33) give the solution of the inhomogeneous model (29) and represent its partition function in the determinantal form.

The explicit answer for the partition function may be obtained if we choose the weight of the vertices

$$
v_{j}=q^{-\frac{j}{2}}, u_{j}=q^{\frac{j-1}{2}}
$$

where $q=e^{-\nu}$, with $\nu>0$ is a Boltzmann weight. Under this parametrization the partition function (27) will take a form

$$
Z(q)=\sum q^{\sum_{k=-1}^{-N} \frac{k}{2}\left(l_{k}^{a}-l_{k}^{b}\right)+\sum_{j=1}^{N} \frac{j-1}{2}\left(l_{j}^{a}-l_{j}^{b}\right)}
$$


where the summation is over all admissible nests of lattice paths. The parametrization (34) transforms (32) into

$$
W_{q}(N, M)=(-1)^{N M+\frac{N(N-1)}{2}}\left\{\prod_{j>k}\left(q^{\frac{j-k}{2}}-q^{-\frac{j-k}{2}}\right)^{-2}\right\} \operatorname{det} Q,
$$

with the matrix elements

$$
Q_{j k}=\frac{s^{\frac{k+j-1}{2}}-s^{-\frac{k+j-1}{2}}}{q^{\frac{k+j-1}{2}}-q^{-\frac{k+j-1}{2}}}
$$

and $s=q^{M-N+2}$. The determinant of the matrix $Q$ was calculated in [7] and is equal to

$$
\operatorname{det} Q=(-1)^{\frac{N(N-1)}{2}}\left\{\prod_{j>k}\left(q^{\frac{j-k}{2}}-q^{-\frac{j-k}{2}}\right)^{2}\right\} \prod_{1 \leq j, k \leq N} \frac{s^{\frac{1}{2}} q^{\frac{j-k}{2}}-s^{-\frac{1}{2}} q^{-\frac{j-k}{2}}}{q^{\frac{k+j-1}{2}}-q^{-\frac{k+j-1}{2}}} .
$$

The substitution of this determinant into (36) gives

$$
\begin{aligned}
W_{q}(N, M) & =(-1)^{N M} q^{-\frac{N^{2}}{2}(M+2-2 N)} \prod_{1 \leq j, k \leq N} \frac{1-q^{M-N+2+j-k}}{1-q^{k+j-1}} \\
& =(-1)^{N M} q^{-\frac{N^{2}}{2}(M+2-2 N)} \prod_{1 \leq j, k \leq N} \frac{1-q^{M+3-j-k}}{1-q^{k+j-1}}
\end{aligned}
$$

and the partition function (35) is given by

$$
Z(q)=q^{-\frac{N^{2}}{2}(M+2-2 N)} \prod_{1 \leq j, k \leq N} \frac{1-q^{M+3-j-k}}{1-q^{k+j-1}} .
$$

From the definition $Z(1)=S(N, M)$ and for the partition function of the homogeneous model (26) we have the following expression

$$
Z\left(\omega_{a}, \omega_{b}, \omega_{c}\right)=\left(\omega_{a} \omega_{b}\right)^{N(M-N+1)} \omega_{c}^{2 N^{2}} \prod_{1 \leq j, k \leq N} \frac{M-j-k+3}{j+k-1} .
$$

To connect the four-vertex model with the tiling model notice that each admissible configuration of lattice paths may be associated with an $N \times N$ array $\pi_{i j}$. The $m$-th path may be thought of as the $m$-th column in this array with a matrix elements $\pi_{j m}$ equal to the number of the cells in the subsequent columns $j$ of the lattice (starting from the right) under the $m$-th path. The array

$$
\pi=\left(\begin{array}{lll}
6 & 4 & 3 \\
5 & 3 & 1 \\
2 & 1 & 0
\end{array}\right)
$$

corresponds to the nest of paths in (FIG. 4). 


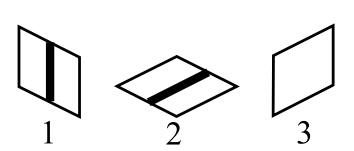

FIG. 5: Three types of lozenges.

An array $\pi_{i j}$ of non-negative integers that are nonincreasing as functions of both $i$ and $j$ $(i, j=1,2, \ldots)$ is called a plane partition $\pi$ [14], the integers $\pi_{i j}$ are the parts of the plane partition. Each plane partition has a three dimensional diagram which can be interpreted as stacks of unit cubes - the three-dimensional Young diagram, $|\pi|=\sum \pi_{i j}$ being its volume. The height of a stack with coordinates $(i, j)$ is equal to the part of plane partition $\pi_{i j}$. If we have $i \leq r, j \leq s$ and $\pi_{i j} \leq t$ for all cubes of the plane partition, it is said that the plane partition is contained in a box with side lengths $r, s, t$. If $\pi_{i j}>\pi_{i+1 j}$, i.e. if the parts of plane partition $\pi$ are decaying along each column, then $\pi$ is called a column strict plane partition. We shall call the strict plane partition the partition $\pi$ that is decaying along each column and each row $\left(\pi_{i j}>\pi_{i+1 j}\right.$ and $\left.\pi_{i j}>\pi_{i j+1}\right)$. The element $\pi_{11}$ of the strict plane partition $\pi_{i j}$ satisfies the condition $\pi_{11} \geq 2 r-2$ if all $i, j \leq r$. An arbitrary plane partition in a box $r \times r \times t$ may be transferred into a strict plane partition in a box $r \times r \times(t+2 r-2)$ by adding to an array $\left(\pi_{i j}\right)$ the matrix

$$
\pi=\left(\begin{array}{cccc}
2 r-2 & 2 r-3 & \cdots & r-1 \\
2 r-3 & 2 r-4 & \cdots & r-2 \\
\vdots & \vdots & & \vdots \\
r-1 & r-2 & \cdots & 0
\end{array}\right)
$$

which corresponds to a minimal strict plane partition.

A plane partition in a $r \times s \times t$ box is equivalent to a lozenge tiling of an $(r, s, t)$-semiregular hexagon. The term lozenge is referred to a unit rhombi with angles of $\frac{\pi}{3}$ and $\frac{2 \pi}{3}$ (FIG. 5 ). The lozenge tilings are simply the projections of three-dimensional diagrams with gradient lines. The three dimensional diagram which corresponds to the plane partition (41) is represented in (FIG. 4).

The partition function of the three dimensional Young diagrams placed into a box is equal to

$$
Z_{Y D}(q)=\sum q^{|\pi|}
$$

where $q$ is a Boltzmann weight and summation is over all plane partitions in a box. The 
volume of the three dimensional strict Young diagram in a box $N \times N \times M$ which corresponds to the allowed arrows (paths) configuration is equal to [17]:

$$
|\pi|=\frac{N^{2} M}{2}+\sum_{k=-1}^{-N} \frac{k}{2}\left(l_{k}^{a}-l_{k}^{b}\right)+\sum_{j=1}^{N} \frac{j-1}{2}\left(l_{j}^{a}-l_{j}^{b}\right) .
$$

Substituting this expression into (35) we obtain the partition function of the three dimensional strict Young diagrams

$$
Z_{Y D}(q)=q^{\frac{N^{2} M}{2}} Z(q)=q^{N^{2}(N-1)} \prod_{1 \leq j, k \leq N} \frac{1-q^{M+3-j-k}}{1-q^{k+j-1}} .
$$

This partition function is the generating function of the strict plane partitions. The number of the correspondent lozenge tilings is $Z_{Y D}(1)$ and is equal to the partition function of the homogeneous model (26) with all weights equal to one:

$$
Z_{Y D}(1)=Z(1,1,1)=\prod_{1 \leq j, k \leq N} \frac{M-j-k+3}{j+k-1} .
$$

\section{PERIODIC BOUNDARY CONDITIONS}

Let us consider now the model on a lattice with the periodic boundary conditions. It means that on a square lattice with $(M+1)$ horizontal rows and $L$ vertical ones the boundary arrows on any horizontal row and on any vertical one are pointing in the same direction (see FIG. 6). Here we shall consider the case when $(M+1)$ and $L$ are even. In the four-vertex
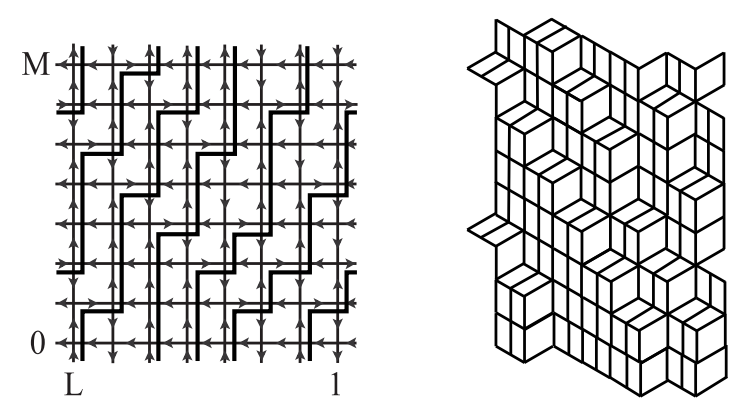

FIG. 6: A typical configuration of arrows and lattice paths for the periodic boundary conditions.

model the number of arrows pointing to the right or to the left is conserved in the subsequent columns of the grid. It means that the number of vertices $c$ per column is also conserved and $l_{c}=2 n$, where $n$ is the number of arrows pointing to the right in a column. 
The partition function (11) of the homogeneous model on a periodic $(M+1) \times L$ lattice with a fixed number of arrows pointing to the right and the vertex weights chosen to be $\omega_{a}=e^{-\nu}, \omega_{b}=e^{\nu}$ and $\omega_{c}=1$ is equal to

$$
Z_{n}(\mu)=\sum e^{\nu\left(l_{b}-l_{a}\right)}
$$

where the summation is carried out over all allowed configurations of arrows on a lattice with the periodic boundary conditions or over all nests of the admissible lattice paths (FIG. $6)$.

Consider the trace of the transfer matrix (10):

$$
\operatorname{tr} \tau^{L}(u)=\operatorname{tr}(A(u)+D(u))^{L}=\sum\left\langle m_{1}, \ldots, m_{n}\left|(A(u)+D(u))^{L}\right| m_{1}, \ldots, m_{n}\right\rangle
$$

Here we denote the state with $n$ spins down in the sites $m_{1}, \ldots, m_{n}$ and with $M+1-n$ spins up in the other sites by $\left|m_{1}, \ldots, m_{n}\right\rangle$, and the sum is taken over the complete set of states $\left|m_{1}, \ldots, m_{n}\right\rangle$ in the quantum space $\mathcal{H}$. The number $n$ is conserved because of the commutativity (14). Since $\tau^{L}(u)$ is a generating function of different combinations of $A$ and $D$ operators, the trace (45) is the sum over all allowed configurations of vertices $(a),(b),(c)$ with the weights $\delta(u), \alpha(u), 1(18)$ on a periodic $(M+1) \times L$ grid

$$
\operatorname{tr} \tau^{L}(u)=\sum(i u)^{l^{b}}\left(i u^{-1}\right)^{l^{a}} .
$$

Comparing this expression with (44) we obtain the following representation for the partition function

$$
Z_{n}(\nu)=e^{-i \frac{\pi}{2}(M+1-2 n) L} \operatorname{tr} \tau^{L}\left(-i e^{\nu}\right)
$$

where we put $u=-i e^{\nu}$.

To calculate this expression one has to solve the eigenvalue problem for the transfer matrix $\tau(u)$ which means finding such a vector $\left|\Psi_{n}\left(v_{1}, \ldots, v_{n}\right)\right\rangle$ that

$$
\begin{aligned}
\tau(u)\left|\Psi_{n}\left(v_{1}, \ldots, v_{n}\right)\right\rangle & =\Theta_{n}\left(u ; v_{1}, \ldots, v_{n}\right)\left|\Psi_{n}\left(v_{1}, \ldots, v_{n}\right)\right\rangle \\
S^{z}\left|\Psi_{n}\left(v_{1}, \ldots, v_{n}\right)\right\rangle & =\frac{1}{2}(M+1-2 n)\left|\Psi_{n}\left(v_{1}, \ldots, v_{n}\right)\right\rangle .
\end{aligned}
$$

The vector $\left|\Psi_{n}\left(v_{1}, \ldots, v_{n}\right)\right\rangle$ is an eigenvector of the transfer matrix if parameters $v_{j}$ satisfy Bethe equations

$$
\begin{aligned}
\left(v_{k}^{2}\right)^{M+1-n} & =(-1)^{n-1} V^{-2}, \quad k=1, \ldots, n . \\
V^{-2} & =\prod_{j=1}^{n} v_{j}^{-2} .
\end{aligned}
$$


By putting $v_{k}^{2}=e^{i p_{k}}$ we can express solutions of the Bethe equations (49) in the form

$$
p_{k}=\frac{2 \pi I_{k}-P}{M+1-n},-\pi<p_{k} \leq \pi
$$

where $I_{n}$ are integers or half-integers depending on $n$ being odd or even, the total momentum $P=\sum_{j=1}^{n} p_{j}$. These solutions were classified in [21, 22].

The eigenvalue of the transfer matrix is equal to

$$
\Theta_{n}(u ;\{v\})=e^{i \frac{\pi}{2}(M+1-2 n)}\left\{u^{M+1} V^{-2}+(-1)^{n} u^{-(M+1-2 n)}\right\} \prod_{j=1}^{n} \frac{1}{u^{2}-v_{j}^{2}} .
$$

The partition function of the four vertex model with the periodic boundary conditions (44) is then given by the equation

$$
Z_{n}(\nu)=e^{-i \frac{\pi}{2}(M+1-2 n) L} \sum_{\{v\}} \Theta_{n}^{L}\left(-i e^{\nu} ;\{v\}\right),
$$

where summation is taken over all different sets of solutions of Bethe equations. The simplest case corresponds to $n=0$. The partition function is $Z_{0}(\nu)=\{2 \cosh (M+1) \nu\}^{L}$, and $Z_{0}(0)=2^{L}$. The other limiting case is when $2 n=M+1$. In this case we have only two sets of solutions. The first is defined by the solutions (50) of Bethe equations with $I_{k}=k-\frac{n+1}{2}$, $k=1, \ldots, n$ and $P=0$. The other one is given by $I_{k}=k-\frac{n+3}{2}, k=1, \ldots, n$, and $P=-\pi$. Applying the equality

$$
\prod_{k=1}^{n}\left(a-b e^{i \frac{2 \pi}{n} k}\right)=a^{n}-b^{n}
$$

one can find that $Z_{\frac{M+1}{2}}(\nu)=2$.

The vector conjugated to the Bethe eigenvector (48)

$$
\left|\Psi_{n}\left(p_{1}, \ldots, p_{n}\right)\right\rangle=\prod_{k=1}^{n} B\left(e^{i p_{k}}\right)|\Leftarrow\rangle,
$$

where $p_{k}$ are the solutions (50) of Bethe equations (49), is equal to

$$
\left\langle\Psi_{n}\left(p_{1}, \ldots, p_{n}\right)\right|=\langle\Leftarrow| \prod_{k=1}^{n} B^{\dagger}\left(e^{i p_{k}}\right) .
$$

The operators $B\left(e^{i p}\right)$ and $C\left(e^{i p}\right)$ are in the involution: $B^{\dagger}\left(e^{i p}\right)=(-1)^{M} C\left(e^{i p}\right)$.

The norm of the Bethe vectors is calculated with the help of (32). When the sets $\{u\}=$ $\{v\}$ the diagonal elements of the matrix $H$ are equal to

$$
H_{j j}=M-n+2
$$


When $\{u\}=\{v\}$ are the solutions of Bethe equations (49) the nondiagonal entries of matrix $H$ are units: $H_{j k}=1$. It is not difficult to calculate the determinant

$$
\operatorname{det} H=(M+1)(M-n+1)^{n-1},
$$

and for the norm of any Bethe eigenvector we obtain:

$$
\mathcal{N}^{2}\left(p_{1}, \ldots, p_{n}\right)=\left\langle\Psi_{n}\left(p_{1}, \ldots, p_{n}\right) \mid \Psi_{n}\left(p_{1}, \ldots, p_{n}\right)\right\rangle=(M+1)(M-n+1)^{n-1} \prod_{j \neq k} \frac{e^{i P}}{e^{i p_{j}}-e^{i p_{k}}}
$$

where $p_{j}$ are the solutions (501). The Bethe vectors (48) form a complete orthogonal set [22].

Consider the cylinder of circumference $M+1$ and the length $L$. The probability that the lattice paths will enter the cylinder at the cites $\tilde{\mu}=\left(\tilde{m}_{1}, \ldots, \tilde{m}_{n}\right) ; \tilde{m}_{i} \geq \tilde{m}_{i+1}+2$ and leave it at the cites $\mu=\left(m_{1}, \ldots, m_{n}\right) ; m_{i} \geq m_{i+1}+2$ is equal to:

$$
\begin{aligned}
& \left\langle m_{1}, \ldots, m_{n}\left|\tau^{L}\left(-i e^{\nu}\right)\right| \tilde{m}_{1}, \ldots, \tilde{m}_{n}\right\rangle \\
= & \mathcal{N}^{-2} \sum_{\{p\}}\left\langle m_{1}, \ldots, m_{n} \mid \Psi_{n}\left(p_{1}, \ldots, p_{n}\right)\right\rangle \Theta_{n}^{L}\left(-i e^{\nu} ;\left\{e^{i p}\right\}\right)\left\langle\Psi_{n}\left(p_{1}, \ldots, p_{n}\right) \mid \tilde{m}_{1}, \ldots, \tilde{m}_{n}\right\rangle \\
= & \mathcal{N}^{-2} \sum_{\{p\}} S_{\mu-\delta}\left(e^{i p_{1}}, e^{i p_{2}}, \ldots, e^{i p_{n}}\right) S_{\tilde{\mu}-\delta}\left(e^{-i p_{1}}, e^{-i p_{2}}, \ldots, e^{-i p_{n}}\right) \Theta_{n}^{L}\left(-i e^{\nu} ;\left\{e^{i p}\right\}\right),
\end{aligned}
$$

the summation is taken over all solutions of Bethe equations, and representation (25) has been used.

We can associate the vertical and horizontal edges of the lattice carrying paths with lozenges. Lozenge (1) in (FIG. 5) corresponds to a vertical line of the path, while lozenge (2) to a horizontal one. Lattice edges without the paths correspond to lozenge (3). The number of lozenge tilings of the torus is given by (52) with $\nu=0$.

\section{CONCLUSION}

Though the considered four-vertex model is a particular case of the six-vertex model the determinantal representation of the partition function of the model with the fixed boundary conditions could not be obtained as a limit because of the absence of the correspondent answer for the six vertex case. The same is true for the representation of the mean value (53) in terms of the symmetric functions.

The quantum Hamiltonian commuting with the transfer matrix of the four-vertex model is the Hamiltonian of the $X X Z$ Heisenberg chain with the infinite anisotropy [21, 22]. The 
approach described in this paper will allow to obtain determinantal representations for the correlation functions of the correspondent quantum model.

\section{ACKNOWLEDGMENTS}

The work was partially supported by the CRDF grant RUMI-2622-ST-04 and RFBR grant 07-01-00358.

[1] R.G. Baxter, Exactly Solved Models in Statistical Mechanics (San Diego, Academic press, 1982)

[2] V.E. Korepin, Calculation of norms of Bethe wave functions, Comm. Math. Phys. 86, 391 (1982).

[3] V. Korepin, P. Zinn-Justin, Thermodynamic limit of the Six-Vertex Model with Domain Wall Boundary Conditions, J. Phys. A 33, 7053 (2000).

[4] N.M.Bogoliubov, A.G.Pronko, M.B.Zvonarev, Boundary correlation functions of the six-vertex model, J. Phys. A 35, 5525 (2002).

[5] D. Allison, N. Reshetikhin, Numerical study of the 6-vertex model with domain wall boundary conditions, Ann. Inst. Fourier(Grenoble) 55, 1847 (2005).

[6] O. Syljuåsen, M. Zvonarev, Directed-loop monte carlo simulations of vertex models, Phys. Rev. E 70, 016118 (2004).

[7] G. Kuperberg, Another proof of the alternating-sign matrix conjecture, Int. Math. Res. Not. 1996, 139 (1996).

[8] F.Colomo, A.G.Pronko, Square ice, alternating sign matrices, and classical orthogonal polynomials, J. Stat. Mech. JSTAT, P01005 (2005).

[9] P.L. Ferrari, H. Spohn, Domino tilings and the six-vertex model at its free fermion, cond-mat/0605406.

[10] W. Li, H. Park, M. Widom, Finite-size scaling amplitudes in a random tiling model, J. Phys. A: Math. Gen. 23, L573 (1990).

[11] W. Li, H. Park, Logarithmic singularity in the surface free energy near commensurateincommensurate transitions, J. Phys. A: Math. Gen. 24, 257 (1991). 
[12] L.D. Faddeev, Quantum Inverse Scattering Method, Sov. Sci. Rev. Math. C1, 107 (1980).

[13] V.E. Korepin, N.M. Bogoliubov, A.G. Izergin, Quantum Inverse Scattering Method and Correlation Functions (Cambridge University Press, Cambridge, 1993).

[14] I. G. Macdonald, Symmetric functions and Hall polynomials, (Clarendon Press, 1995).

[15] D.M. Bressoud, Proofs and Confirmations. The Story of the Alternating Sign Matrix Conjecture, (Cambridge University Press, Cambridge, 1999).

[16] A. Vershik, Statistical mechanics of combinatorial partitions and their limit configurations, Funct. Anal. Appl. 3090 (1996).

[17] N.M. Bogoliubov, Boxed plane partitions as an exactly solvable boson model, J. Phys. A 38, 9415 (2005).

[18] N. Tsilevich, Quantum Inverse Method for the q-boson model and symmetric functions, Funct. Anal. Appl. 40, 53 (2006).

[19] K. Shigechi, M. Uchiyama, Boxed skew plane partition and integrable phase model, J. Phys. A: Math. Gen. 38, 10287 (2005).

[20] N.M. Bogoliubov, Enumeration of plane partitions and the algebraic Bethe ansatz, Theor. Math. Phys. 150, 165 (2007).

[21] M. Gaudin, La Fonction d'onde de Bethe, (Masson, Paris, 1983).

[22] N.I. Abarenkova, A.G. Pronko, The temperature correlator in the absolutely anisotropic Heisenberg XXZ-magnet, Theoret. and Math. Phys. 131, 690 (2002). 